\title{
Flexibility windows and compression of monoclinic and orthorhombic silicalites
}

\author{
Asel Sartbaeva* \\ Department of Chemistry, Inorganic Chemistry Laboratory, South Parks Road, Oxford, OXI 3QR, United Kingdom, European Union \\ Julien Haines and Olivier Cambon \\ Institut Charles Gerhardt Montpellier, UMR 5253 CNRS-UM2-ENSCM-UM1, Equipe C2M, Université Montpellier 2, Place E. Bataillon, \\ cc 1504, F-34095 Montpellier, Cedex 5, France, European Union \\ Mario Santoro \\ Ist. Fis. Applicata Nello Carrara (IFAC)-CNR, Via Madonna del Piano, 10, I-50019 Sesto Fiorentino (Florence), Italy and European \\ Laboratory for non Linear Spectroscopy (LENS), Via Nello Carrara,1, Sesto Fiorentino (Florence), Italy, European Union \\ Federico Gorelli \\ LENS, Via Nello Carrara, 1, I-50019 Sesto Fiorentino (Florence), Italy, European Union and CNR, Ist Proc Chim Fis, \\ Unita Org Supporto Roma, I-00185 Rome, Italy, European Union \\ Claire Levelut \\ Laboratoire Charles Coulomb, UMR 5221 CNRS, Université Montpellier 2, Place E. Bataillon, cc069, F-34095 Montpellier, \\ Cedex 5, France, European Union \\ Gaston Garbarino \\ European Synchrotron Radiation Facility (ESRF), F-38343 Grenoble, France, European Union \\ Stephen A. Wells \\ Department of Physics and Centre for Scientific Computing, University of Warwick - Gibbet Hill Road, Coventry CV4 7AL, \\ United Kingdom, European Union
}

(Received 12 September 2011; revised manuscript received 16 January 2012; published 13 February 2012)

\begin{abstract}
Silicalite, a high-silica zeolite with MFI topology, undergoes pressure-induced amorphisation (PIA) when compressed with nonpenetrating pressure media. PIA is prevented by penetrating pressure media. In several zeolites, links have recently been found between pressure-induced phase transitions and the flexibility window, a geometric property of the framework identified by geometric simulation. We have analyzed structural data from compression experiments on silicalite, and we find that PIA occurs while the structure lies within its flexibility window. Penetrating media, which prevent PIA, push the structure outside the flexibility window. Thus framework flexibility is required for PIA to occur in silicalite. This link between amorphisation and flexibility is further evidence of the deep connections between framework geometry, flexibility, and the physical properties of zeolites.
\end{abstract}

DOI: 10.1103/PhysRevB.85.064109

PACS number(s): 61.50.Ks, 61.43.Gt, 81.05.Rm, 82.33.Jx

\section{INTRODUCTION}

The aluminosilicate zeolites are a diverse group of minerals with complex and beautiful microporous framework structures. ${ }^{1}$ Many zeolites have found industrial applications as ion-exchange materials and as shape- and size-selective catalysts and molecular sieves, due to their specific pore and channel geometries and variable framework composition., $^{2,3}$ As a result of their use, particularly as industrial catalysts, zeolite structures have been mainly studied at high temperatures, and there have been relatively few detailed structural studies of zeolites under high-pressure conditions. Modeling and simulation can extract additional insight and information from the available experimental data. Recent combined experimental-theoretical investigations of zeolites ${ }^{4-9}$ have revealed deep links between the geometry and the physics of zeolite frameworks. In this study, we investigate the pressure-induced amorphisation of a siliceous zeolite structure in the context of framework flexibility.
Silicalite is an almost pure silica $\left(\mathrm{SiO}_{2}\right)$ zeolite with the MFI framework topology. ${ }^{1}$ Monoclinic silicalite under ambient conditions becomes orthorhombic, and then metrically tetragonal, under pressure. ${ }^{10}$ With nonpenetrating pressure media, the structure undergoes gradual pressure-induced amorphisation (PIA) at pressures of 3-8 GPa, possibly with some rebonding but retaining much of the original network topology. With penetrating media $\left(\mathrm{CO}_{2}, \mathrm{Ar}\right)$, the framework does not become amorphous at pressures up to $25 \mathrm{GPa}$, as the channel content prevents collapse of the framework. ${ }^{10}$ Under slow compression, the bulk modulus of silicalite at ambient conditions $(13.6 \mathrm{GPa})$ is low and becomes even lower $(9.9 \mathrm{GPa})$ at pressures above $3 \mathrm{GPa} ;{ }^{11}$ under rapid compression, a value of $18.8 \mathrm{GPa}$ has been obtained. ${ }^{10}$ This indicates a very high capacity for framework flexibility. The use of a penetrating pressure transmitting medium induces a higher bulk modulus: 35.9 GPa, comparable to quartz, showing direct compression of the framework.

The technique of geometric simulation, a simplified method based on polyhedral templates, has been successfully applied 
to elucidate the physics of manganite ${ }^{12,13}$ and zeolite ${ }^{4-9}$ frameworks. Recent studies have shown that zeolite frameworks display a geometric property termed a "flexibility window." "Over a range of densities, it is in principle possible for the framework to be made geometrically ideal; that is, the structural tetrahedra can in principle have perfectly regular geometry without distortions or steric clashes. Outside the window, distortions of the polyhedra are geometrically inevitable, even in the absence of long-range interactions, host-guest interactions, and other physical effects.

The interatomic interactions in a zeolite structure can be divided into two components, $U_{\text {local }}$ and $U_{\text {nonlocal }}$. $U_{\text {local }}$ contains those interactions which favor ideal tetrahedral geometry and steric exclusion, while $U_{\text {nonlocal }}$ contains all other interactions, including those between the framework and the channel contents. The geometric simulation effectively models $U_{\text {local }}$ while neglecting $U_{\text {nonlocal }}$. If the structure lies within its flexibility window, then it can reach the minimum of $U_{\text {local }}$. The introduction of $U_{\text {nonlocal }}$ will cause some distortion of the $\mathrm{SiO}_{4}$ units from the tetrahedral geometric ideal, but this distortion represents variation around a minimum. Its energy penalty in the $U_{\text {local }}$ terms will be small and second order in the size of the distortion. If on the other hand the structure lies outside its flexibility window, it cannot reach the minimum of $U_{\text {local }}$. Any further distortion of the framework will come at an energy penalty which is large and first order in the size of the distortion. We therefore expect the structure to behave differently in, for example, the interactions between the framework and channel contents, depending on whether it lies inside or outside its flexibility window.

In the analcime group of very dense aluminosilicate zeolites, ${ }^{7-9}$ the flexibility window is intimately linked to pressure-induced phase transitions. The structures tend to remain in their high-symmetry forms so long as they can remain within the flexibility window; when the limits of the flexibility window are reached, they transform to very low-symmetry forms. We wish to investigate links between the flexibility window and pressure-induced phase transitions of other zeolite systems. The available data on silicalite permit us to study the links between the flexibility window of a framework and its behavior in the presence or absence of channel content. The almost pure silica composition of the material, and the use of a nonionic pressure transmitting medium, maximizes the significance of steric effects compared to ionic interactions.

Detailed refinement of the distribution of channel content under pressure was not possible, and so we lack direct information on the interactions between the framework and channel content. Without such information, the application of empirical-potential or $a b$ initio simulations to study the structure in compression would be challenging, if not impossible. However, geometric simulation can reveal whether such interactions affect the framework flexibility.

\section{METHOD}

High-pressure x-ray diffraction experiments on silicalite-1$\mathrm{OH}$ were performed with diamond anvil cells on the beam line ID27 at the ESRF as described previously. ${ }^{10}$ Cell parameters have been obtained for silicalite under compression both with and without a penetrating pressure transmitting medium, ${ }^{10,11}$ and are given in Tables I and II. Atomic coordinates for silicalite under ambient conditions were also obtained from Olson et al. ${ }^{14}$ Without a penetrating medium, the structure undergoes gradual PIA, and so cell parameters obtained at higher pressures must be treated with caution, as they are likely to be nonequilibrium values and possibly subject to local depressurization effects. ${ }^{11}$ For this study, we assume these parameters represent the crystalline portions of a mixed crystalline/amorphous phase.

The extent of the flexibility window for silicalite is assessed using geometric simulation. ${ }^{4-6}$ Cell parameters appropriate to each $P-V$ data point were applied to a unit cell of the MFI framework. The simulation cell contains all framework atoms explicitly in P1 symmetry. All runs began with a small random perturbation, of magnitude $0.05 \AA$, to all atomic positions. The program "GASP" then relaxes the framework, seeking to make each $\mathrm{SiO}_{4}$ unit into an ideal tetrahedron with an Si-O bond length of $1.61 \AA$, and to avoid steric overlap between oxygen atoms using a steric radius of $1.35 \AA .{ }^{1}$ The details of geometric simulation in GASP have been previously described. ${ }^{4,5,15}$ Geometric relaxation proceeds by steepestdescent minimization of the mismatch between atoms and tetrahedral templates. The residual mismatch then represents deformations of the geometry of the bonded group away from the geometric ideal defined by the template (Fig. 1). There are two possible outcomes: One is that the framework is left

TABLE I. $P-V$ data for compression of silicalite with a nonpenetrating pressure transmitting medium (silicone oil), and data for two fictitious structures testing the effect of the ortho/mono transition on framework flexibility. $a / c$ ratios are given for each structure; where the structure is not metrically tetragonal, the $a / c$ ratio is calculated using the average of the very similar $a$ and $b$ parameters, and is marked with an asterisk. In the final column, structures are annotated with a $\mathrm{Y}$ if they can be made stress-free in geometric simulation, that is, the tetrahedra can in principle be made geometrically ideal.

\begin{tabular}{lccccccc}
\hline \hline$P(\mathrm{GPa})$ & $a(\AA)$ & $b(\AA)$ & $c(\AA)$ & $\beta\left(^{\circ}\right)$ & $\mathrm{V}\left(\AA^{3}\right)$ & $a / c$ & Stress-free \\
\hline 0 & 19.895 & 20.125 & 13.379 & 90.629 & 5356.26 & $1.495^{*}$ & $\mathrm{Y}$ \\
0.4 & 19.773 & 19.955 & 13.294 & 90.460 & 5245.29 & $1.494^{*}$ & $\mathrm{Y}$ \\
1.1 & 19.583 & 19.728 & 13.174 & 90 & 5089.47 & $1.492^{*}$ & $\mathrm{Y}$ \\
3.46 & 19.224 & 19.224 & 12.700 & 90 & 4693.43 & 1.514 & 1.521 \\
4.54 & 18.969 & 18.969 & 12.473 & 90 & 4488.07 & $\mathrm{Y}$ \\
5.5 & 18.992 & 18.992 & 12.716 & 90 & 4586.38 & 1.494 & $1.494^{*}$ \\
0.4 ortho & 19.773 & 19.955 & 13.294 & 90 & 5245.45 & $\mathrm{Y}$ \\
1.1 mono & 19.583 & 19.728 & 13.174 & 90.35 & 5089.38 & $1.492^{*}$ \\
\hline \hline
\end{tabular}


TABLE II. $P-V$ data for compression of silicalite with $\mathrm{CO}_{2}$ pressure medium $(3.45-21.2 \mathrm{GPa})$ and with Ar pressure medium (6.8-22.2 GPa), and data for three fictitious structures testing the significance of the cell volume and a/c ratio for framework flexibility. $a / c$ ratios are given for each structure; where the structure is not metrically tetragonal, the $a / c$ ratio is calculated using the average of the very similar $a$ and $b$ parameters, and is marked with an asterisk. In the final column, structures are annotated with an $\mathrm{N}$ if they cannot be made stress-free in geometric simulation, that is, the tetrahedra cannot in principle be made geometrically ideal. In the last three cases, the $a$ and $b$ parameters are taken from an experimental structure (pressures of 4.54, 5.5, and 7.3 GPa). The $c$ parameters are adjusted to control the $a / c$ ratio. These three cases are marked with $\dagger$.

\begin{tabular}{|c|c|c|c|c|c|c|}
\hline$P(\mathrm{GPa})$ & $a(\AA)$ & $b(\AA)$ & $c(\AA)$ & $V\left(\AA^{3}\right)$ & $a / c$ & Stress-free \\
\hline 3.45 & 19.381 & 19.456 & 13.154 & 4959.99 & $1.476^{*}$ & $\mathrm{~N}$ \\
\hline 7.3 & 18.874 & 18.874 & 12.832 & 4570.85 & 1.471 & $\mathrm{~N}$ \\
\hline 10.3 & 18.564 & 18.564 & 12.583 & 4336.41 & 1.475 & $\mathrm{~N}$ \\
\hline 13.3 & 18.37 & 18.37 & 12.490 & 4215.00 & 1.471 & $\mathrm{~N}$ \\
\hline 15.7 & 18.207 & 18.207 & 12.364 & 4098.67 & 1.473 & $\mathrm{~N}$ \\
\hline 17.3 & 18.196 & 18.196 & 12.341 & 4085.89 & 1.475 & $\mathrm{~N}$ \\
\hline 17.7 & 18.173 & 18.173 & 12.323 & 4069.54 & 1.475 & $\mathrm{~N}$ \\
\hline 21.2 & 17.996 & 17.996 & 12.203 & 3951.98 & 1.475 & $\mathrm{~N}$ \\
\hline 6.8 & 19.005 & 19.005 & 12.843 & 4638.84 & 1.480 & $\mathrm{~N}$ \\
\hline 8.5 & 18.846 & 18.846 & 12.754 & 4529.82 & 1.478 & $\mathrm{~N}$ \\
\hline 10.5 & 18.694 & 18.694 & 12.540 & 4382.33 & 1.491 & $\mathrm{~N}$ \\
\hline 13.3 & 18.505 & 18.505 & 12.398 & 4245.70 & 1.492 & $\mathrm{~N}$ \\
\hline 17.52 & 18.110 & 18.110 & 12.289 & 4030.59 & 1.474 & $\mathrm{~N}$ \\
\hline 22.2 & 17.856 & 17.856 & 12.064 & 3846.46 & 1.480 & $\mathrm{~N}$ \\
\hline- & 18.992 & 18.992 & $12.920^{\dagger}$ & 4660.19 & 1.469 & $\mathrm{~N}$ \\
\hline- & 18.969 & 18.969 & $12.819^{\dagger}$ & 4612.57 & 1.479 & $\mathrm{~N}$ \\
\hline- & 18.874 & 18.874 & $12.583^{\dagger}$ & 4482.33 & 1.500 & $\mathrm{~N}$ \\
\hline
\end{tabular}

stress-free by the geometric relaxation, with all atoms lying within a small tolerance $(0.001 \AA)$ of ideal tetrahedral geometry; the other is that the framework cannot reach tetrahedral geometry, and is left stressed. The results of our simulations are reported in Tables I and II; each data point is annotated with a Y if the framework is left stress-free, i.e., it lies within its flexibility window, or with an $\mathrm{N}$ if it is left stressed, i.e., it lies outside its flexibility window.

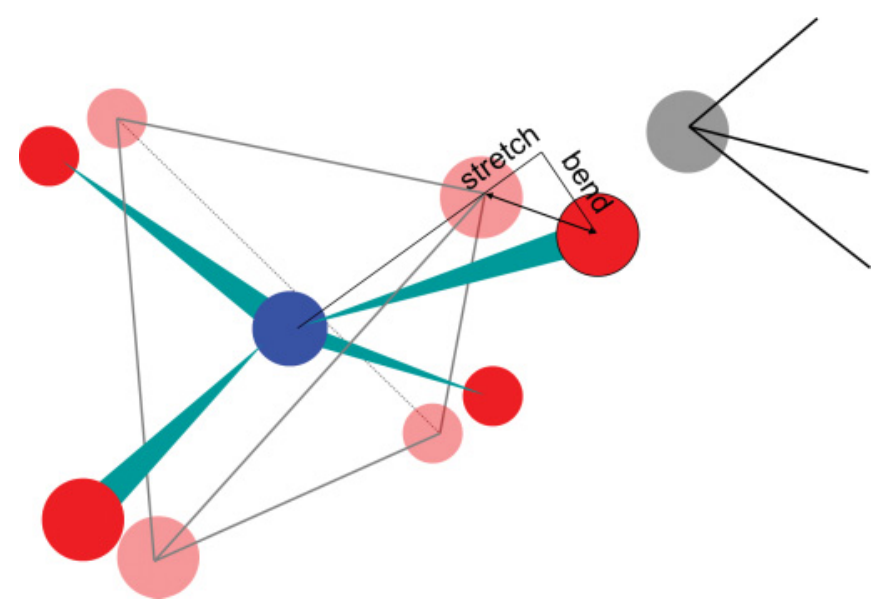

FIG. 1. (Color online) In geometric simulation, the mismatch between atoms (blue-T atom, red-vertex atom; dark grey-in print) and templates is decomposed into components of bond-stretching, parallel to the bond vector in the template, and of bond-bending, perpendicular to the bond vector in the template. Template is outlined by lines between template atoms (light grey).
Examples of the silicalite framework after geometric relaxation with various cell parameters are shown in Fig. 2. The structures in Figs. 2(a) and 2(b) are stress-free in geometric relaxation; the structures in Figs. 2(c) and 2(d) cannot be fully relaxed, and contain intrinsic stress.

\section{RESULTS AND DISCUSSION}

\section{A. Compression without a penetrating pressure transmitting medium}

In Table I, we present cell parameters from compression without a penetrating pressure transmitting medium, annotated with the results of geometric simulation. The monoclinic frameworks at pressures of $0 \mathrm{GPa}$ and $0.4 \mathrm{GPa}$ relax, and so lie within the flexibility window. Orthorhombic frameworks at pressures $1.1-5.5 \mathrm{GPa}$, in the course of PIA, ${ }^{16}$ are also relaxable and lie within the flexibility window. In contrast to the case for analcime, ${ }^{9}$ in silicalite the first phase transitionfrom monoclinic at $0.4 \mathrm{GPa}$ to orthorhombic at $1.1 \mathrm{GPa}$-is not associated with the edge of the flexibility window, as the framework remains stress-free in geometric simulation on either side of the transition. To investigate this transition further, we have tested two additional, nonexperimental sets of cell parameters. In one set, $a, b$, and $c$ are taken from the experimental monoclinic structure at $0.4 \mathrm{GPa}$, but the beta angle is set to 90 degrees to give an orthorhombic structure. In another, $a, b$, and $c$ are taken from the experimental orthorhombic structure at $1.1 \mathrm{GPa}$, and the beta angle is set above 90 degrees to give a monoclinic structure. These sets are also given in Table I as "0.4ortho" and "1.1mono." Both sets are 


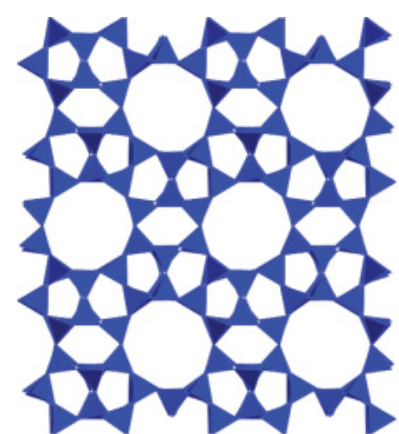

(a)

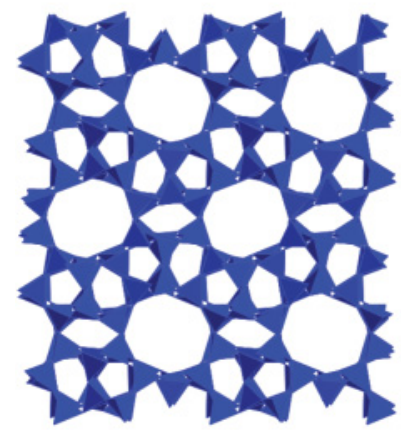

(c)

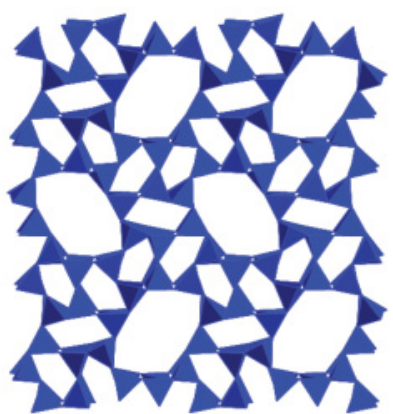

(b)

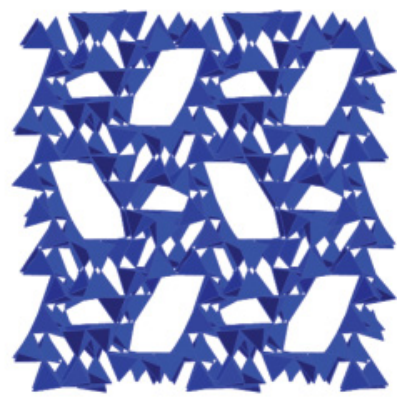

(d)
FIG. 2. (Color online) Geometrically simulated silicalite frameworks using cell parameters taken from different compression states: (a) under ambient conditions; (b) at $5.5 \mathrm{GPa}$ with nonpenetrating pressure media; (c) at $3.45 \mathrm{GPa}$ with $\mathrm{CO}_{2}$; d) at $17.7 \mathrm{GPa}$ with $\mathrm{CO}_{2}$.

relaxable, confirming that the orthorhombic and monoclinic forms of MFI have overlapping flexibility windows.

\section{B. Compression with penetrating pressure media}

In Table II, we present cell parameters from compression with penetrating media $\left(\mathrm{CO}_{2}\right.$ and $\left.\mathrm{Ar}\right)$ annotated with the results of geometric simulation. In this case, the structure does not become amorphous, remaining crystalline at pressures up to $22 \mathrm{GPa}$. We find that none of the frameworks are geometrically relaxable. The effect of the penetrating media is apparently to push the framework out of its flexibility window and into an intrinsically stressed state. It is striking that this introduction of stress into the framework prevents the more substantial framework alteration involved in pressure-induced amorphisation.

\section{Significance of the $a / c$ ratio}

The range of cell volumes in Table II, with the structure outside the flexibility window, overlaps with the range of cell volumes in Table I, with the structure inside the flexibility window. However, the situations can be distinguished by the value of the $a / c$ ratio. In the overlapping range of volumes (above $4488 \AA^{3}$ ), the structures which relax have $a / c$ ratios above 1.49 , whereas those which do not relax have ratios of 1.48 or below. It thus appears that the effect of the penetrating pressure transmitting medium within the channels of the structure is to over-extend the $c$ axis, and so force the structure

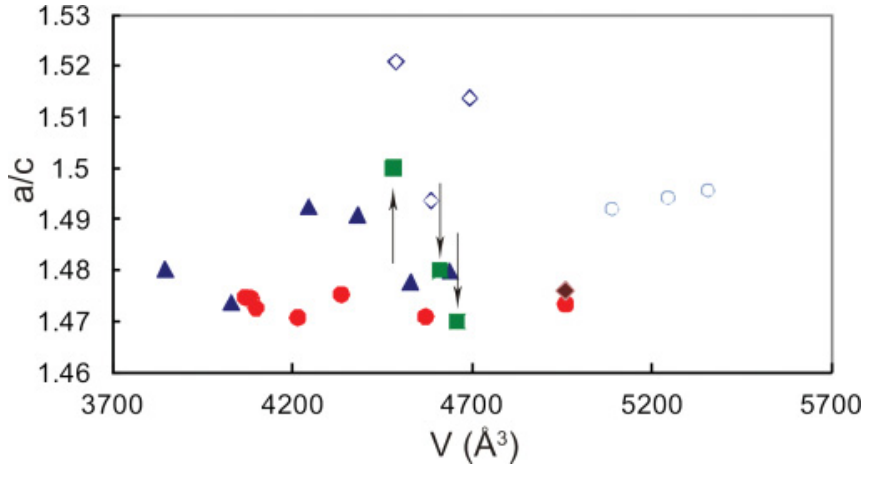

FIG. 3. (Color online) $a / c$ ratio vs cell volume for structures reported in Table I and II. Open symbols indicate data within the flexibility window, and filled symbols indicate data outside the window. Three nonexperimental points are shown as green squares and indicated with arrows showing the direction in which their $a / c$ ratios have been changed; red filled circles and brown filled diamond-structures with $\mathrm{CO}_{2}$; blue filled triangles—structures with Ar; other-structures without penetrating medium.

out of its flexibility window. The limits of the flexibility window of the metrically tetragonal structure, as a function of cell volume and $a / c$ ratio, are summarized in Fig. 3 .

To confirm the significance of the $a / c$ ratio to the limits of the flexibility window, we consider two additional sets of nonexperimental cell parameters, given in Table II. In these cases, the $a$ parameter is taken from a relaxable structure in Table I (pressures of 4.54 and $5.5 \mathrm{GPa}$ ), and the $c$ parameter is chosen so as to give an $a / c$ ratio below 1.48 . This change increases the cell volume but prevents the structure from relaxing.

This naturally raises the question of whether a structure obtained during compression with a penetrating pressure transmitting medium would become relaxable with a higher $a / c$ ratio. We therefore consider an additional set of nonexperimental cell parameters at the end of Table II, taking the $a$ parameter from the structure at a pressure of $7.3 \mathrm{GPa}$ and reducing the $c$ parameter to give an $a / c$ ratio of 1.5 . This structure, however, still does not relax to become stress-free under geometric simulation. We have thus found limits on the flexibility window of the orthorhombic MFI framework in both cell volume and cell shape ( $a / c$ ratio). The cell volume of this structure lies slightly below those of the relaxable structures in Table I. This indicates that the framework could not be further compressed beyond the range explored in Table I without passing out of its flexibility window; in other words, pressure-induced amorphisation takes place while the structure is within its flexibility window.

The $P-V$ data are summarized in Fig. 4. The pressurevolume data were fitted to a Birch-Murnaghan equation of state (EOS): ${ }^{17}$

$$
\begin{aligned}
P= & 1.5 B_{0}\left[\left(V / V_{0}\right)^{-7 / 5}-\left(V / V_{0}\right)^{-5 / 3}\right] \\
& \times\left[1+0.75\left(B_{0}^{\prime}-4\right)\left(\left[V / V_{0}\right]^{-2 / 3}-1\right)\right],
\end{aligned}
$$

where $V_{0}, B_{0}$, and $B_{0}^{\prime}$ are the volume, bulk modulus, and its derivative at ambient pressure, respectively. The parameters for silicalite, as reported previously, ${ }^{10}$ are $B_{0}=18.8 \mathrm{GPa}$, $B^{\prime}=4.0$, without penetrating pressure transmitting medium. 


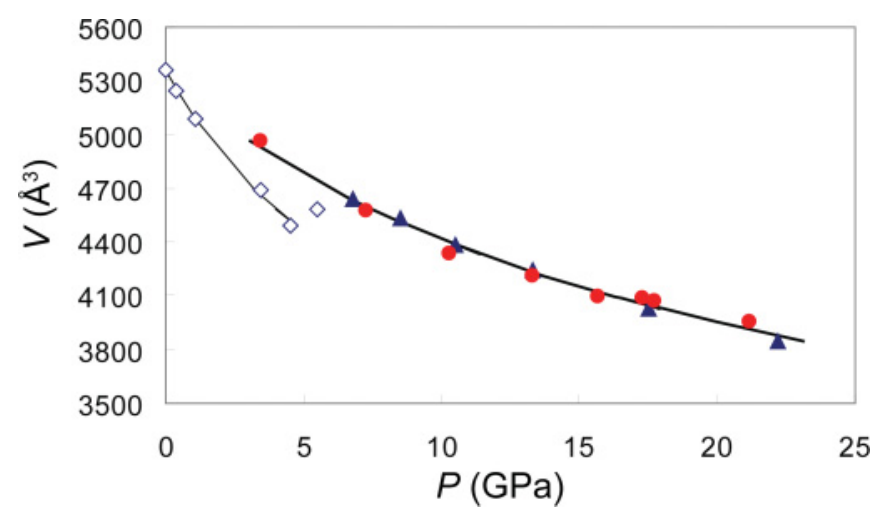

FIG. 4. (Color online) Pressure vs cell volume for silicalite with [circles $\left(\mathrm{CO}_{2}\right)$, triangles (Ar)] and without (diamonds) penetrating pressure medium. Open symbols indicate data within the flexibility window and filled symbols indicate data outside the window. The lines are fits to a second-order Birch-Murnaghan EoS.

A least-squares fit to the combined $\mathrm{CO}_{2} / \mathrm{Ar}$ data sets gives $B_{0}=35.5 \mathrm{GPa}, B^{\prime}=4.0$.

\section{CONCLUSIONS}

We have applied geometric simulation to silicalite frameworks over a wide range of cell parameters obtained from compression experiments with and without a penetrating pressure transmitting medium. In addition, we have considered several sets of nonexperimental cell parameters constructed to test the nature of the phase transitions in the structure. For each set of cell parameters, we have determined whether or not the structure can be made stress-free by geometric relaxation and thus lie within its flexibility window.

In compression with a nonpenetrating pressure transmitting medium, i.e., with an empty framework, the structure remains relaxable through the monoclinic to orthorhombic phase transition and through the onset of pressure-induced amorphisation at pressures from 3 to $5.5 \mathrm{GPa}$. Upon further compression, the structure reaches the limit of its flexibility window. Thus it appears that PIA proceeds while the structure lies within its flexibility window, and is complete once the structure is compressed beyond the limit of the window. In compression with a penetrating pressure transmitting medium, which occupies the channels and prevents collapse of the framework, the structure is forced out of its flexibility window by overextension of the $c$ parameter, and is not relaxable at any point in the compression.

These results provide evidence that the framework tends to remain within its flexibility window as far as possible, and leaves the flexibility window only when forced to do so by excessive external pressure or internal pressure from the introduction of channel content. This provides further evidence of the link between the geometric and physical properties of zeolite structures. ${ }^{4,9}$ It also appears that the progress of pressure-induced amorphisation in silicalite depends on the crystalline portions of the structure remaining within their flexibility window, as it is the flexibility of the framework which allows for folding under compression.

\section{ACKNOWLEDGMENTS}

A.S. thanks the Royal Society for funding, S.A.W. thanks the Leverhulme foundation for funding, and J.H. thanks the French National Research Agency (ANR) for funding through the research project ANR-09-BLAN-0018-01. We acknowledge support from the European Union (European Laboratory for Nonlinear Spectroscopy Contract FP7 G.A. No. 228334 LASERLABEUROPE) and the Ente Cassa di Risparmio di Firenze. *asel.sartbaeva@chem.ox.ac.uk

${ }^{1}$ C. Baerlocher, A. Hepp, and W. M. Meier, DLS76, a FORTRAN program for the simulation of crystal structures by geometric refinement, Institut fur Kristallographie und Petrographie, ETH, Zurich, Switzerland (1978).

${ }^{2}$ D. Breck, Zeolites Molecular Sieves (John Wiley and Sons, Inc., New York, 1974).

${ }^{3}$ S. Kulprathipanja, Zeolites in Industrial Separation and Catalysis (Wiley-VCH, Weinheim, 2010).

${ }^{4}$ A. Sartbaeva, S. Wells, M. Treacy, and M. Thorpe, Nat. Mater. 12, 962 (2006).

${ }^{5}$ G. D. Gatta and S. A. Wells, Phys. Chem. Miner. 31, 1 (2004).

${ }^{6}$ G. D. Gatta and S. A. Wells, Phys. Chem. Miner. 33, 243 (2006).

${ }^{7}$ A. Sartbaeva, G. Gatta, and S. A. Wells, Europhys. Lett. 83, 26002 (2008).

${ }^{8}$ G. D. Gatta, A. Sartbaeva, and S. A. Wells, Eur. J. Mineral. 21, 571 (2009).
${ }^{9}$ S. A. Wells, A. Sartbaeva, and G. Gatta, Europhys. Lett. 94, 56001 (2011).

${ }^{10}$ J. Haines, O. Cambon, C. Levelut, M. Santoro, F. Gorelli, and G. Garbarino, J. Am. Chem. Soc. 132, 8860 (2010).

${ }^{11}$ J. Haines, C. Levelut, A. Isambert, P. Hébert, S. Kohana, D. Keen, T. Hammouda, and D. Andrault, J. Am. Chem. Soc. 131, 12333 (2009).

${ }^{12}$ A. Sartbaeva, S. A. Wells, M. F. Thorpe, E. S. Bozin, and S. J. L. Billinge, Phys. Rev. Lett. 97, 065501 (2006).

${ }^{13}$ A. Sartbaeva, S. A. Wells, M. F. Thorpe, E. S. Božin, and S. J. L. Billinge, Phys. Rev. Lett. 99, 155503 (2007).

${ }^{14}$ D. H. Olson, G. T. Kokotailo, S. L. Lawton, and W. M. Meier, J. Phys. Chem. 85, 2238 (1981).

${ }^{15}$ S. A. Wells, M. T. Dove, and M. G. Tucker, J. Phys. Condens. Matter 14, 4567 (2002).

${ }^{16}$ G. N. Greaves, F. Meneau, A. Sapelkin, L. M. Colyer, I. ap Gwynn, S. Wade, and G. Sankar, Nat. Mater. 2, 622 (2003).

${ }^{17}$ F. Birch, Phys. Rev. 71, 809 (1947). 